\title{
Commonalities and Differences Between the Chinese Quantifier "Zhang" and the Japanese Quantifier "Mei" in Cognitive Category
}

\author{
Menghan Tian
}

School of Law, Humanities and Sociology, Wuhan University of Technology, Wuhan 430063, China

*Corresponding author. Email: m13155836632@163.com

\begin{abstract}
In Chinese, "Zhang"(张) is used as a quantifier to describe flat objects, which is similar to the quantifier "Mei"(枚). Besides, they have many other different usages. Summarizing the commonalities and differences from the modified objects, it is found that they have similar categorization principles, and their prototype members have different extent expansion under the driving factors. The similarity of principles reflects the commonality of human cognition of language, while the specific differences reflect the diversity of human civilization.
\end{abstract}

Keywords: Quantifier, Prototype members, Categorization, Motivation.

\section{INTRODUCTION}

"Mei" (枚) is often used in Japanese to describe things with planar sense, such as paper, photo, etc, which is similar to "Zhang"(张), a quantifier in Chinese. Through the enumeration analysis of the objects they modified, it is found that the specific objects modified by them are quite different, but they are consistent with the principle of categorization. Both Chinese and Japanese classifiers belong to the category words. In this case, both of them should conform to the general laws of human language category words [1].
We can explore the commonality of the categorical principles of the Chinese quantifier "Zhang" (张) and the Japanese quantifier "Mei" (枚), through analyzing their prototype members, sorting out the developmental scope expansion, and researching the motivation of the scope expansion.

Combining with the research of Yan Du (2010) and Shanshan Cao (2019), on the basis of searching Chinese and Japanese vocabulary, the commonalities and differences of the two quantifiers are compared as follows:

Table 1. Comparison of the use of Chinese quantifier "Zhang" and Japanese quantifier "Mei"

\begin{tabular}{|c|c|c|c|}
\hline & & Zhang ( 张) & Mei ( 枚 ) \\
\hline & Generality & Measure some flat objects & Measure some flat objects \\
\hline Category & Difference & $\begin{array}{l}\text { Measure the face of a person or } \\
\text { animal } \\
\text { Measure something that can be } \\
\text { opened and closed }\end{array}$ & $\begin{array}{l}\text { Weigh slender objects } \\
\text { Counting the number of actors, } \\
\text { geisha, sumo wrestler, etc. and other } \\
\text { ordinary people } \\
\text { Measurement ranking order }\end{array}$ \\
\hline \multirow[b]{2}{*}{ Individual } & Generality & $\begin{array}{l}\text { Paper, paper money, ticket, } \\
\text { photo, visiting card, fur, cushion, mat, } \\
\text { Spier }\end{array}$ & $\begin{array}{l}\text { Paper, paper money, ticket, } \\
\text { photo, visiting card, fur, cushion, mat, } \\
\text { Spier }\end{array}$ \\
\hline & \multicolumn{3}{|c|}{ Table 1, cont } \\
\hline
\end{tabular}




\begin{tabular}{|l|l|l|l|}
\hline & Difference & Bed, mouth, bow, net, face, lyre & $\begin{array}{l}\text { Feather, coin, clothing, board, } \\
\text { wall, light, the number of ordinary } \\
\text { people }\end{array}$ \\
\hline
\end{tabular}

"paper", "paper money", " board", "leaf", "feather" and so on. Such as, 一枚の紙 (a piece of paper), 一枚の葉 (a leaf), 写真一枚 (a photo) .The more peripheral members are clothes, wall, the number of ordinary people, etc.

\section{PROTOTYPE MEMBERS}

Cognitive psychologist Rosch believes that none of the attribute is necessary while distinguishing a category. "The categorization of entities is based on good and clear samples, and then other entities are classified into this category based on their similarities with them in some or a set of attributes."[2] In short, the prototype is the most typical and representative member of a conceptual category. The commonality between the prototypes of the Chinese quantifier "Zhang" (张) and the Japanese quantifier "Mei" (枚) is that they are both used to describe things with flat surfaces.

The prototype members of "Zhang"(张) are things with planar as the main feature, such as paper and photo. Determining the members of the prototype category can be carried out in the following three aspects: First, from a diachronic level, there is "bow", "plow" and "lyre". The original meaning of "Zhang" (张 ) is to open the bow string to prepare for launch, and the other is to hang the string of the bow, both interpretations are directly related to the "bow". Because the plow and the lyre are similar to the bow's shape. According to the principle of cognitive similarity, it summarizes similar things as the same category, and modifies them with "Zhang" (张). Secondly, the psychological reality level, which is the member that comes to mind first when people mention it. Taylor (1989 cited from Yan Du 2010) proposed that the typical has psychological reality and listed several possible causes: (1) Human beings are born with the characteristics of perception; (2) Typical cases are common; (3) Typical cases are learned earlier; (4) Typical has the average value of cases with this attribute; (5) The significance of attributes; (6) Typical has a higher categorization benefit. When talking about "Zhang" (张), you will think of things such as "paper" and "photo" first, instead of "face" or "mouth". Therefore, "paper" and "photo" belong to the prototype members in the category. Thirdly, the cognitive level, prototype members should have specific and simple characteristics, abstract and complex things are at the edge of it. For example, "paper" and "table" are specific and common things with obvious characteristics, so they belong to prototype members. Inversely, the "face" is an abstract thing derived from the evolution of cognition on the basis of archetypes.

The prototype members of "Mei" (枚)are also flat things, specifically, they are flat and thin things, like

\section{CATEGORY EXPANSION}

Although from the prototype members to the margin expansion, the specific objects in the category expansion of the Chinese quantifier "Zhang"(张) and the Japanese quantifier "Mei" (枚) are quite different, there are similarities in principle. Then we will discuss it in detail, from three aspects and explores the commonality of its category principles.

\subsection{Extend from a Two-dimensional Plane to Another that Contains the Whole Three- Dimensional Thing}

Everything is three-dimensional. The abovementioned "two-dimensional planar" means that the third dimension of things is very small in scale, which can be ignored.

Yuzhi Shi (2001) believes that Chinese twodimensional spatial quantifiers include "Tiao" (条) and "Zhang"(张), with $\mathrm{XY}$ as the dimension and $\mathrm{X}$ as longer dimension. When the value of $\mathrm{Y} / \mathrm{X}$ is close to 1 or equal to 1, we will use "Zhang"(张), or use "Tiao"(条), when approaching 0. Based on that, paper, photo and cushion are using" Zhang"(张). The most common usage of the Japanese quantifier "Mei" (枚) is also to describe similar flat and thin things, such as: a piece of paper, a photo a photo, and two cushions.

Because of the small proportion of the third dimension, the above examples can be roughly understood as two-dimensional flat things, which are common and the most typical specific things, and belong to prototype members in the category of modified objects of "Zhang" (张) and "Mei" (枚), who emphasized their prominent planar features. "Zhang" ( 张) modifies things such as bed, table, and stool. Using "Zhang" to measure them, because the "planar" in the configuration of these things is the most functional part in the process of interaction with humans. The twodimensional plane is the most prominent feature. [3]The Japanese quantifier "Mei" (枚) can modify things that are not two-dimensional plane, such as "feather" and "board", which also captures their flat and thin feature. "Mei" (枚) can also modify "field" and "wall", who are not two-dimensional plane, nor do they conform to 
people's perception of "thinness", but the plane is still the prominent feature of people's intuitive feeling.

From this point of view, both the Chinese quantifier "Zhang"(张) and the Japanese quantifier "Mei" (枚) have metaphors and metonymy that substitute features for the whole. However, the specific metaphor and metonymy contents are different, and we will make a detailed analysis later.

\subsection{Expanded from Visual Features to Functional Features}

The original meaning of "Zhang" (张) in Chinese is closely related to "bow", which refers to the action of pulling a bow. Visually, the trajectory of the string drawn away from the bow in the air constitutes a plane, so the prototype members are things modified by "Zhang" (张) and with planar as their typical feature. Research on cognition has shown that one of the laws of human cognition is the principle of similarity, that is, treating similar things as things in the same category [4]. "Plow" and "bow" have similar structures and strings, so they also use "Zhang" (张)to measure. According to the visual opening and closing action of pulling the bow, it can be analogized to things like "mouth" and "net", what need to be opened and closed when using. These visual intuitions are expanded to the functional level, such as a bank card, CD, record, etc., who visually present planar features, and the main functions are also concentrated on it. A series of new things have emerged with the development of the times. Things with flat features and functions concentrated on this flat surface are also measured by "Zhang".

The Japanese quantifier "Mei" (枚) can be used to measure the number of inferior people such as a sedanchair bearer, actor, geisha, and sumo wrestlers. Such as: 八枚肩の駕籠 (a palanquin of eight shoulders). The names of professionals such as actors, geishas, sumo wrestlers, etc. will be written on boards or posters, bulletin boards, etc., which are all flat and thin objects, and measured by the quantifier "Mei" (枚). After that "Mei" (枚) is also used to measure these people. Then from measuring these specific people to measuring the number of ordinary people [5].

\subsection{Expand from the Concrete Plane to the Abstract Plane}

The more specific, direct, and typical members of the cognitive category are located at the center of the category, otherwise the more abstract and implicit members are located at the periphery of the category. "Zhang"(张) can modify "脸" ( face), or in more abstract terms: "面孔" ( face, but it doesn't exactly correspond to the word "face.", also meaning "Countenance" ) . Zhang can be used to measure the fur of animals, such as a mink. Animal fur also has the characteristics of flatness. After the scope is expanded, it can be used not only to measure animal fur, but also to measure human skins. After being blurred, it can be used to measure "脸" and "面孔".

"Mei" (枚) was initially used to measure painting, poster, and board, after blurring it can be used to measure the geisha, actor, and sumo wrestler published on them. Besides, after the number of people was blurred, it was further blurred to measure these geisha and sumo wrestler's ranking. Therefore, the Japanese quantifier "Mei" (枚) can also be used for grades ranking.

Both "Zhang" (张)and "Mei" (枚)have gone through the process of blurring in the development of using, they are used to describe abstract plane extending from concrete plane or abstract things related to plane. However, due to the difference in the scope, direction, and the specific blurring methods, the two quantifiers have greatly deviated in the selection of the modified objects.

\section{MOTIVATION}

\subsection{Metaphor and Metonymy}

Metaphor generally recognizes the unfamiliar, invisible, abstract, and rare concept domains from familiar, tangible, concrete, and common one, thus establish connections between different conceptual systems [6].

"Zhang"(张) originally means to open the bow and is used as a quantifier for the bow. Based on that, because the plow and the lyre are similar to the bow in appearance, so it is a metaphor to describe them. According to the characteristics that the bow can be opened, it is also used to describe other things that can be opened and closed, such as mouth and fishing net.

The prototype members of "Mei" (枚) are also flat objects, but they can be used to describe "feather" or "shell". Although they are different from regular flat objects such as "paper" or "painting", they are also flat and thin. So it can be summarized into the category of "Mei"(枚)

Metonymy, like metaphor, is an important cognitive model. Metaphor is the mapping between different domains, while metonymy is to replace inconspicuous things with prominent features in the same domain, or replace the whole with more prominent parts [7].

The metonymy of "Zhang" (张) can be summarized into the following two types: the first is using the partial to metonymy the whole, such as "table", "bed" and "bench". The plane is its distinctive feature. Therefore, although the whole is presented as a cube, it can also 
use "Zhang" (张) instead of "Kuai"(块). The second is using content to metonymy container, such as "a slogan" and "a notice". "Slogan" and "notification" are texts, which are abstract things without specific outlines. So they can't use quantifiers that used to describe the shape. However, in people's perception, slogans are written on typical flat objects such as notice, advertisement, newspaper ect., whose text is the focus of attention. Therefore, in the field of cognition, in order to facilitate the description and measurement, directly use the quantifier of its container "Zhang" (张) to measure the content written on it.

The Japanese quantifier "Mei"(枚), from the perspective of using partial to metonymy the whole, it is used to measure "board", "land", and "wall", that's because we are focusing on their remarkable planar features, and then metaphors the whole thing. From the perspective of the content metonymy container, the usage of "Mei" (枚) to measure geisha, actor, and sumo wrestler is based on their names and portraits on posters, bulletin board, and other containers. Like the content metonymy containers of "Zhang" (张), it is focused on the content, then expanding from the names and portraits of real people, finally to the entire group.

\subsection{Image Schema}

According to Zhiming Qin's opinion, the image schema of "Zhang" (张) can be divided into two types: "planar schema" and "opening schema". "Planar schema", that is to say, in people's cognition, the first reaction is its plane feature, such as "table", "bed", and "record". "Open schema" refers to measuring something that can be opened and closed, such as "mouth" and "net", when mentioning mouth we will associate with its opening and closing movements, and thinking of retracting and releasing of the net when talking about it.

"Mei" (枚) can also be explained by "planar schema", such as "wall", "field" and other things with planar as their prominent feature. You can also use container icons to explain the usage of "Mei"'s measurement of geisha, sumo wrestler, actor, etc., whose names and portraits appear on bulletin board and posters that serve as containers, and then refer to the person himself.

\section{CONCLUSIONS}

The Chinese quantifier "Zhang" (张) and the Japanese quantifier "Mei" (枚) have the same prototype member: an item characterized by a plane. And they have similar categorization principles: the expansion of cognitive categories is achieved through metaphor and partial metonymy, while the content metonymy is the containers of the whole. However, through enumeration and analysis, it is found that there is a big difference in the selection of specific measurement objects, which is because Japanese has evolved its own language characteristics due to the influence of factors, such as the characteristics of the national culture and the individual learners [8], which are unpredictable and uncontrollable. As Shouyun Zong said: the consistency of principles reflects the common cognitive methods of mankind, and the difference in details reflects the diversity of human attributes.

\section{REFERENCES}

[1] Zong Shouyun. The principle commonality and details of the cognitive category: Taking the similarities and differences between the Chinese quantifier "tiao" and the Japanese quantifier "Ben" as an example. [J] Rhetorical Learning, Issue 2, 2011

[2] Renshun Jin (Korea). A study of the Selectional Relationships of the Shape-based Classifiers with Noums in Chinese $[\mathrm{J}]$. Doctoral dissertation, Shanghai Normal University, May 2019

[3] Yalan Wen. Teaching Polysemy Based on Prototype Theory. [J]Journal of Nanjing Xiaozhuang University, 2006 (3)

[4] Yan Du. Semantic Cognitive Analysis of the Measure Word "Zhang".[J] Overseas Chinese Education. Phase II, 2010

[5] Shanshan Cao. A comparative study of Chinese and Japanese Quantifier "Mei" [J]Journal of Kunming University, 2019, 41 (5): $125 \sim 132$

[6] Ying Wang. Cognitive Linguistics. [M] Shanghai Foreign Language Education Press. 2007

[7] Zhiming Qin. The Research of Modern Chinese Quantifier. [J] Shenyang Normal University. 2015

[8] Xiaole Lv. The Phenomenon of Language Transfer from Chinese to Japanese_-Taking Quantifiers as an Example [J]Modern Society 2021, Issue 2 